\title{
ANÁLISIS FILOGENÉTICO DE XENARTROS (MAMMALIA) BASADOS EN ELEMENTOS ÓSEOS DEL APARATO HIOIDES: ASPECTOS SOBRE LA MONOFILIA DE GLIPTODÓNTIDOS
}

\author{
MARTÍN ZAMORANO \\ División Paleontología de Vertebrados, Museo de La Plata, Facultad de Ciencias Naturales y Museo, Universidad Nacional \\ de La Plata, CONICET. Cátedra de Zoología Vertebrados. Paseo del Bosque s/n, 1900 La Plata, Argentina. \\ marzamorano@fcnym.unlp.edu.ar
}

\begin{abstract}
Phylogenetic analysis of Xenarthra (Mammalia) based on bone elements of the hyoid apparatus: aspects on the monophyly of glyptodontids. The morphology and disposition of the bone elements of the hyoid apparatus preserved in fossil mammals allow us to elaborate hypotheses about how the tongue participates in obtaining, processing and swallowing the food. Traditionally, in the phylogenetic analyses of Xenarthra, dental, skeletal and molecular characters have been used. In this contribution, a phylogenetic analysis was performed using exclusively hyoid apparatus characters. The possible importance of these characters in the phylogeny of xenarthrans is discussed here. A second analysis is carried out, forcing the monophyly of the large traditional xenarthra groups. We also review aspects of the monophyly of glyptodontids. A matrix of 15 taxa and 30 characters of the hyoid apparatus was constructed, nine modified from the existing literature and 21 constitute an original contribution. The analyses were carried out with the TNT program, through an exhaustive search. In the first analysis, a maximum parsimony tree was obtained $(\mathrm{L}=60),(\mathrm{CI}=0.75),(\mathrm{IR}=0.87)$, from which the Bremer and Bootstrap support values were calculated. In this first analysis, all xenarthrans are recovered as a natural group. However, despite considering the monophyly of the glyptodontids there are some discrepancies in the relationships of large groups of xenarthrans. In the second analysis, forcing some clades to be monophyletic, one maximum parsimony tree was obtained $(\mathrm{L}=77),(\mathrm{CI}=0.58),(\mathrm{IR}=0.72)$. Glyptodontidae is the only natural group with taxonomic status present in both analyses; it is also the clade of Xenarthra with closer phylogenetic linkage and greater reliability.
\end{abstract}

Keywords: Glyptodontidae, Panochthus, phylogeny, Glyptodon, hyoid apparatus.

RESUMO - A morfologia e disposição dos elementos ósseos do aparelho hioide preservados em mamíferos fósseis, permitem elaborar hipóteses a respeito de como a língua participa na obtenção, processamento e deglutição do alimento. Tradicionalmente, nas análises filogenéticas dos xenartros, são utilizados caracteres dentários, esqueléticos e moleculares. O que se discute aqui é a possível relevância do uso destes caracteres na filogenia dos xenartros. Uma segunda análise foi feita, forçando o monofiletismo dos grandes grupos tradicionais dos xenartros. Também foi revisado aspectos sobre o monofiletismo dos gliptodontes. Foi construida uma matriz com 15 táxons e 30 caracteres referentes ao aparelho hioide, sendo que nove modificados da literatura existente e 21 originais. As análises foram feitas com o programa TNT, através de uma busca exaustiva. Na primeira análise foi recuperada uma árvore de máxima parcimônia $(\mathrm{L}=60),(\mathrm{IC}=0,75),(\mathrm{IR}=0,87)$, da qual foi calculado os índices de suporte de Bremer e Bootstrap. Nesta primeira análise foi recuperada uma topologia com todos xenartros como grupo natural. Apesar de se considerar a monofilia dos gliptodontes, existem algumas discrepâncias em relação aos grandes grupos de xenartros. Na segunda análise, forçando o monofiletismo, se obteve uma árvore de máxima parcimônia $(L=77),(I C=0,58),(I R=0,72)$. Gliptodontidae foi o único grupo natural presente em ambas as análises e também foi o clado de xenartros com vinculação filogenética mais próxima e com maior confiabilidade.

Palavras-chave: Glyptodontidae, Panochthus, filogenia, Glyptodon, aparelho hioide. 


\section{INTRODUCCIÓN}

La condición de grupo natural de los Xenarthra está claramente sustentada, en tanto que los caracteres tradicionalmente utilizados para definir las sinapomorfías clásicas de este grupo son morfológicos, basados principalmente en elementos esqueletarios y dentarios (Engelmann, 1985; Gaudin, 1995, 1999, 2004; Gaudin et al., 1996, McDonald, 2003; Rose et al., 2005; Gaudin \& McDonald, 2008). Asimismo los estudios filogenéticos con base en datos moleculares también sostienen que este es un grupo monofilético (de Jong et al., 1985; Sarich, 1985; Delsuc et al., 2001, 2002, 2003; Madsen et al., 2001; Murphy et al., 2001); en años recientes se publicaron análisis que incluyen algunos de los taxones aquí analizados (tardígrados fósiles y gliptodóntidos), basados en ADN antiguo (ADNa) y colágeno (Buckley et al., 2015; Delsuc et al., 2016; Slater et al., 2016).

Los xenartros constituyen un muy particular grupo de mamíferos característico de la Región Neotropical, ampliamente representado en el registro fósil de América del Sur, tanto por su extensión temporal como por su frecuencia de registros (Paula Couto, 1979; Scillato-Yané, 1982; Wetzel, 1982; Scillato-Yané \& Carlini, 1995; Zamorano, 2012). Puede afirmarse que prácticamente no existe ninguna unidad estratigráfica continental fosilífera del Cenozoico argentino que no sea portadora de restos de xenartros (Rusconi, 1937; ver Ameghino, 1889, 1906; Kraglievich, 1934; Scillato-Yané, 1982, 1986). La gran mayoría de los taxones de xenartros se registran en América del Sur (Paleoceno superior-Reciente) (Scillato-Yané, 1976, 1982; Vizcaíno et al., 1990; ScillatoYané et al., 1995). En cuanto a su diversidad, el grupo estuvo representado en el pasado por más de 160 géneros fósiles reconocidos (Hoffstetter, 1958, 1982; McKenna \& Bell, 1997) y probablemente con más de 400 especies (ver Mones, 1986), los cuales se suceden durante casi todo el Cenozoico; actualmente el clado incluye 31 especies vivientes agrupadas en 14 géneros (Wetzel, 1985; Redford \& Eisenberg, 1992; McKenna \& Bell, 1997). La gran diversificación del grupo se ve reflejada, entre otras, en la amplia gama de dietas y de modos de adquirir el alimento. Estas incluyen herbívoros, omnívoros, carnívoros (Vizcaíno \& De Iuliis, 2003; Vizcaíno et al., 2004; McDonald, 2005; Gaudin \& Croft, 2015). Tradicionalmente, Xentarhra se divide, principalmente, en dos grandes grupos: Pilosa (incluyendo Vermilingua y Tardigrada) y Cingulata (Wetzel, 1985; Redford \& Eisenberg, 1992; McKenna \& Bell, 1997). Dentro de los Cingulata, la familia Glyptodontidae es un grupo extinto de xenartros acorazados característicos de América del Sur (Eoceno superior-Holoceno Inferior), cuyos representantes alcanzaron un gran tamaño, a veces gigantesco (Scillato-Yané \& Carlini, 1998; Fariña, 2001; Zamorano et al., 2014), pudiendo superar los $2.300 \mathrm{~kg}$ (Soibelzon et al., 2012). Panochthus Burmeister, 1866 y Glyptodon Owen, 1938 son de los gliptodóntidos más abundantes y diversificados del Pleistoceno sudamericano; asimismo, son de los que poseen mayor abundancia de registros en la Región Pampeana (Hoffstetter, 1958; Paula Couto, 1979; Scillato-Yané et al., 1995; Cione et al., 1999;
Soibelzon et al., 2006; Zamorano, 2012; Zamorano et al., 2014; Zurita et al., 2016). Estos son los gliptodóntidos de mayor distribución latitudinal y altitudinal en América del Sur (Zurita et al., 2009, 2016; Zamorano, 2012). También en estos últimos dos géneros incluyen especies que están dentro de los cingulados de mayor tamaño (ver Fariña, 2001).

En los mamíferos se encuentra entre las ramas mandibulares y articulando con el cráneo el aparato hiodes, el cual se forma a partir de los cartílagos del segundo y tercer arco branquial que se osifican tempranamente (König \& Liebich, 2005). En los ejemplares adultos generalmente se encuentra formado por 10 elementos óseos y 2 elementos cartilaginosos asociados. Los 10 huesos son: (1) estilohioides (par), (2) epihioides (par), (3) ceratohioides (par), (4) tirohioides (par) y (5) basihioides (impar). En el curso de la ontogenia, los basihioides izquierdo y derecho se fusionan formando una barra transversal llamada "cuerpo del hioides". En tanto que, en los xenartros se fusionan (4) y (5) para formar el hueso impar ( $V$-bone) (Flower, 1885; Jayne, 1898; Taylor \& Weber, 1951; Naples, 1986; Shoshani \& Marchant, 2001; Pérez et al., 2010; Casali \& Perini, 2016; Zamorano et al., 2018). Los dos cartílagos mencionados son: (a) tímpanohioides y (b) condrohioides, los cuales no se conocen enmamíferos fósiles (ver Zamorano et al., 2018). Particularmente en mamíferos fósiles resulta interesante hacer inferencias sobre cómo se relacionaría el aparato hioides con la lengua para colaborar con la forma de alimentarse (Pérez et al., 2010; Zamorano et al., 2018).

En esta contribución se lleva a cabo un análisis filogenético a base de caracteres extraídos de los elementos óseos del aparato hioides. Esto implica en sí mismo un análisis parcializado en cuanto a las verdaderas relaciones filéticas de los grupos incluidos. Se discute aquí la importancia de dichos caracteres en la filogenia de los xenartros. La obtención de grupos coherentes con filogenias previas, anteriormente citadas, permiten destacar la importancia de caracteres que podrían utilizarse para definir estos grupos. De igual modo se efectúa un segundo análisis forzando la monofilia de los grandes grupos y se revisan aspectos sobre la monofilia de gliptodóntidos.

\section{MATERIAL Y MÉTODOS}

Se estudió la variación morfológica del aparato hioides en 15 taxones de mamíferos placentarios, siete vivientes y ocho fósiles (vide infra). Los materiales de gliptodóntidos se observaron de manera directa; en tanto que la información sobre los restantes taxones fue obtenida mediante la revisión de la literatura específica para cada grupo (Burmeister, 1864, 1871, 1874; Scott, 1905; Gasc, 1967; Sisson \& Grossman,1977; Naples, 1986, 1999; Reiss, 1997; Pérez et al., 2000, 2010; König \& Liebich, 2005; Casali \& Perini, 2016) (ver Apéndice 1).

Los materiales que en esta contribución se observaron directamente son: (1) MPLK 0004, un cráneo muy completo con ambas ramas mandibulares, el aparato hioides completo con todos sus elementos pares e impares y el cartílago tiroides osificados, y el escudete cefálico. Procede del Pleistoceno 
Superior de Marcos Paz. Museo Paleontológico "Lucas Kraglievich", Marcos Paz, Buenos Aires, Argentina (MPLK). Atribuido a Panochthus sp. por Zamorano et al. (2018); (2) MHM-P 87, parte del cráneo con las mandíbulas fragmentadas, el hueso impar y fragmentos de los huesos pares del aparato hioides y restos de anillos faríngeos, ambos fémures y ambos húmeros, tibia-fibulay radio-ulna, varios elementos de los tarsos y carpos, tubo dorsal y lumbar, costillas cervicales, y la coraza dorsal casi completa. Procede del Pleistoceno Superior de General Belgrano. Museo Histórico Municipal "Alfredo Enrique Mulgura", General Belgrano, Buenos Aires, Argentina (MHM). Atribuido a Panochthus sp. por Zamorano et al. (2018); (3) MHM-P 34. Cráneo completo con las dos ramas mandibulares, aparato de hioides casi completo y con parte del cartílago tiroides osificado, restos del esqueleto postcraneal,y varios osteodermos de la coraza dorsal. Procede del Pleistoceno Superior de General Belgrano. Atribuido a Glyptodon cf. G. clavipes por Pérez et al. (2000).

Para la terminología anatómica se siguió a Zamorano et al. (2018). Los detalles sobre el análisis cladístico utilizados en este trabajo se exponen en el apartado siguiente.

\section{DESCRIPCIÓN DEL ANÁLISIS FILOGENÉTICO}

Se construyó una matriz de 15 taxones y 30 caracteres (Apéndice 2). Felis silvestris catus Linneo, 1758, un felino doméstico del cual se ha estudiado con detalle el aparato hioides y cuyos elementos son representativos de los de un mamífero generalizado, fue utilizado como grupo externo para enraizar el árbol. Se incluyeron dos grandes herbívoros introducidos en la llanura Pampeana: Equus ferus caballus Linneo, 1758 y Bos primigenius taurus Linneo, 1758; a su vez, también se incluyeron representantes de todos los grupos de xenartros (2 Vermilingua, Myrmecophaga tridactyla Linneo, 1758 y Tamandua tetradactyla Linneo, 1758; 2 tardígrados vivientes, Bradypus variegatus Schinz, 1825 y Choloepus hoffmanni Peters, 1858; 2 tardígrados fósiles, Glossotherium robustum Owen, 1842 y Megatherium americanum Cuvier, 1796; 2 armadillos vivientes, Priodontes maximus (Kerr, 1792) y Dasypus novemcinctus Linneo, 1758; 2 armadillos fósiles, Proeutatus sp. y Prozaedyus sp. Las especies de gliptodóntidos del mismo género se reunieron en dos grupos, (a) grupo Panochthus [incluye los materiales atribuidos a Panochthus sp. (MPLK 0004 y MHM-P 87), y los atribuidos por Burmeister (1874) a P. tuberculatus (Owen, 1845)], y (b) grupo Glyptodon (incluye el material asignado a Glyptodon cf. G. clavipes Owen, 1839 (MHM-P 34), y los atribuidos por Burmeister (1874) a G. reticulatus Owen, 1845, G. clavipes Owen, G. elongatus Burmeister, 1866).

Los 30 caracteres utillizados mayoritariamente son nuevos, 9 modificados de la literatura (1 de Gaudin, 2004; 4 de O'Leary et al., 2013 y 4 de Casali \& Perini, 2016, ) y 21 constituyen un aporte original del presente trabajo. Hay 17 caracteres binarios y 13 multiestado (series de transformación no ordenadas) (ver Apéndice 2). Los estados de carácter que no se pudieron codificar aparecen como "?". La matriz se elaboró a través del programa Mesquite, versión 3.10 (Maddison \& Maddison, 2016).

Los análisis se efectuaron con el programa TNT (Tree analysis using New Technology) 1.1 (Goloboff et al., 2008a) y se realizó una búsqueda exhaustiva a través de la opción "implicit enumeration". Un segundo análisis se realizó forzando la monofilia de los Pilosa (Myrmecophaga tridactyla, Tamandua tetradactyla, Bradypus variegatus, Choloepus hoffmanni, Glossotherium robustum y Megatherium americanum), Tardigrada (Bradypus variegatus, Choloepus hoffmanni, Glossotherium robustum y Megatherium americanum) y Cingulata (Priodontes maximus, Dasypus novemcinctus, Proeutatus sp., Prozaedyus sp., grupo Panochthus y grupo Glyptodon) a través de la opción "Define Constraints" del TNT (Goloboff et al., 2008b). De todos los árboles se calculó la longitud - pasos - (L), el Índice de Consistencia (IC) e Índice de Retención (IR).

\section{RESULTADOS}

En el primer análisis se obtuvo un árbol de máxima parsimonia con las siguientes características: $(L=60)$, $(\mathrm{IC}=0,75),(\mathrm{IR}=0,87)$, del cual se calcularon los valores de soporte Bremer (Goloboff \& Farris, 2001) y de Bootstrap (Soltis \& Soltis, 2003) (Figura 1). En este primer análisis se separan los ungulados y se recuperan a todos los xenartros como un grupo natural apoyado por ocho sinapomorfías: carácter 1(1), carácter 2(1), carácter 3(1), carácter 4(1), carácter 9(1), carácter 12(1), carácter 13(1), carácter 26(1), carácter 27(1) (ver Tabla 1); por su parte los ungulados (Equus ferus caballus y Bos primigenius taurus) forman un grupo monofilético sostenido por dos sinapomorfías: carácter 22(1), carácter 24(1). Sin embargo, y a pesar de recuperar a los gliptodóntidos (grupo Panochthus + grupo Glyptodon) como un grupo natural soportado por ocho sinapomorfías: carácter 1(3), carácter 4(3), carácter 7(2), carácter 8(1), carácter 14(1), carácter 22(1), carácter 26(2), carácter 28(1) (ver Tabla 1), y tener como grupo hermano a Proeutatus sp., este último clado está apoyado por cinco sinapomorfía: carácter $5(1)$, carácter 10(1), carácter $15(1)$, carácter 25(2), carácter 29(2) (ver Tabla 1), existen algunas discrepancias en las relaciones filogenéticas de los grandes grupos de xenartros.

A partir del segundo análisis se obtuvo un árbol de máxima parsimonia ( $\mathrm{L}=77),(\mathrm{IC}=0,58),(\mathrm{IR}=0,72)$ (Figura 2$)$, en donde el clado Xenarthra está sustentado por diez sinapomorfías: carácter 1(1), carácter 2(1), carácter 3(1), carácter 4(1), carácter $7(0)$, carácter $9(1)$, carácter $12(1)$, carácter $13(1)$, carácter 26(1), carácter 27(1). Cabe destacar que este análisis incluye una sinampomorfía más que en el análisis previo (ver Tabla 1). A su vez, los Xenarthra se separan del clado que conforman los ungulados (Equus ferus caballus y Bos primigenius taurus), que presenta las mismas sinapomorfías que en el análisis anterior. La monofilia forzada de los Cingulata está sustentada solo por una sinapomorfía: carácter 30(2). En este análisis, el grupo natural de los gliptodóntidos (grupo Panochthus + grupo Glyptodon) está soportado por nueve sinapomorfías: carácter $7(2)$, carácter $8(1)$, carácter 


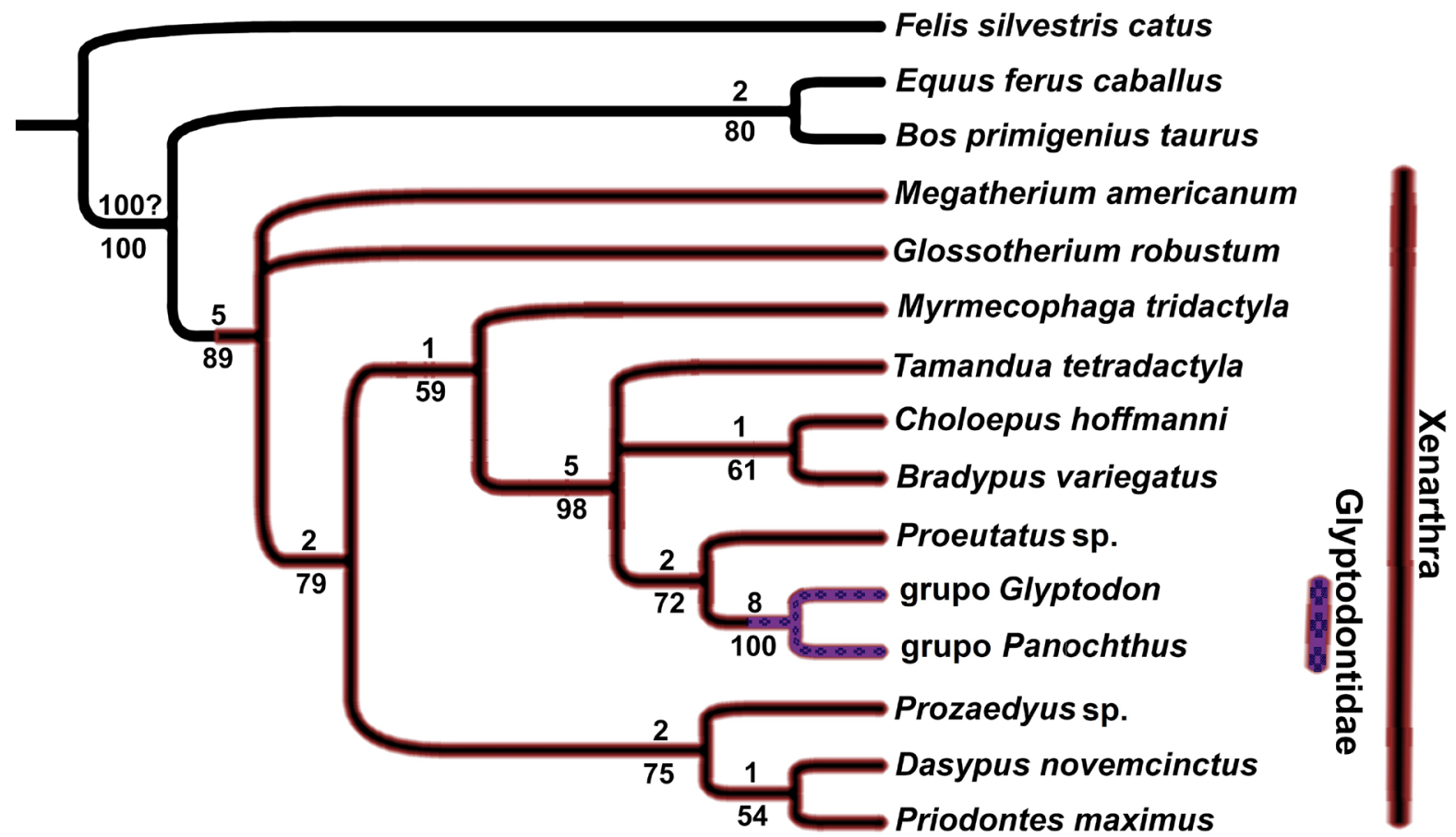

Figura 1. Árbol más parsimonioso. Los números indican el valor del soporte de Bremer (por encima de la rama) y del soportes de Bootstrap (por debajo de la rama).

Figure 1. Most parsimonious tree, the numbers indicate the Bremer support value (above branch) and Bootstrap support (below branch).

Tabla 1. Comparación de las sinapomorfías de los análisis con enumeración implícita (L:60) y forzando la monofilia (L:77). Se destacan los sinapomorfías de nodos comunes.

Table 1. Comparison of the synapomorphies of the analyzes with implicit enumeration (L: 60) and forcing monophyly (L: 77). There are highlighted the synapomorphies of common nodes.

\begin{tabular}{|c|c|c|}
\hline ÁRBOL & NODO & SINAPOMORFIAS \\
\hline \multirow{3}{*}{ Enumeración implícita (L:60) } & Xenarthra & $1(1), 2(1), 3(1), 4(1), 9(1), 12(1), 13(1), 26(1), 27(1)$ \\
\hline & $\begin{array}{l}\text { grupo Panochthus } \\
\text { + grupo Glyptodon }\end{array}$ & $1(3), 4(3), 7(2), 8(1), 14(1), 22(1), 26(2), 28(1)$ \\
\hline & $\begin{array}{l}\text { (grupo Panochthus } \\
\text { + grupo Glyptodon) } \\
\text { + Proeutatus sp. }\end{array}$ & $\mathbf{5}(\mathbf{1}), \mathbf{1 0}(1), \mathbf{1 5}(\mathbf{1}), 25(2), \mathbf{2 9}(2)$ \\
\hline \multirow{7}{*}{$\begin{array}{l}\text { Monofilia forzada } \\
\text { (L:77) }\end{array}$} & Xenartrha & $1(1), 2(1), 3(1), 4(1), 7(0), 9(1), 12(1), 13(1), 26(1), 27(1)$ \\
\hline & $\begin{array}{l}\text { grupo Panochthus } \\
\text { + grupo Glyptodon }\end{array}$ & 7(2), 8(1), 11(1), 14(1), 21(1), 22(1), 26(2), 27(2), 28(1) \\
\hline & $\begin{array}{l}\text { (grupo Panochthus } \\
\text { + grupo Glyptodon) } \\
\text { + Proeutatus sp. }\end{array}$ & 5(1), 6(1), 10(1),15(1), 29(2) \\
\hline & Cingulata & $30(2)$ \\
\hline & Pilosa & sin sinapomorfias \\
\hline & Vermilingua & $23(1), 30(1)$ \\
\hline & Tardigrada & sin sinapomorfías \\
\hline
\end{tabular}


11(1), carácter 14(1), carácter 21(1), carácter 22(1), carácter 26(2), carácter 27(2), carácter 28(1), una sinapomorfía más que en el anterior análisis (ver Tabla 1). También, como en el primer análisis, Proeutatus sp. es su grupo hermano y en este caso también está soportado por cinco sinapomorfías: carácter $5(1)$, carácter $6(1)$, carácter $10(1)$, carácter $15(1)$, carácter 29(2), pero no son las mismas que en el primer análisis (ver Tabla 1). Las monofilias forzadas de Pilosa y de Tardigrada no están apoyadas por ninguna sinapomorfía (ver Tabla 1). Dentro del clado de los Tardigrada existen dos agrupaciones monofiléticas; aquella que incluye los Tardigrada fósiles está sustentada por una sinapomorfía: carácter 18(1); la otra agrupación está formada por los tardígrados vivientes y está soportada por siete sinapomorfías: carácter 3(2), carácter $6(1)$, carácter 11(1), carácter 21(1), carácter 27(2), carácter 29(1). Cabe destacar que esta última agrupación monofilética también aparece en el primer análisis y allí está sostenido por tres sinapomorfías: carácter 20(0), carácter 25(1), carácter $30(0)$. Por último, la monofilia forzada de Vermilingua está sustentada por dos sinapomorfías: carácter 23(1), carácter 30(1).

En ambos análisis, el clado de los xenartros es el que está sustentado por más sinapomorfías, seguido por el de los gliptodóntidos; de este último grupo, el $66 \%$ de las sinapomorfías son compartidas en los dos análisis (Figura 3) (ver Tabla 1).

\section{DISCUSIÓN}

De las aproximadamente 400 especies de xenartros fósiles que se han reconocido (ver Mones, 1986) se registran restos pertenecientes al aparato hioides solo de 20 taxones [Analcimorphus giganteus Ameghino, 1893 (Scott, 1905;
Pérez et al., 2010), Eremotherium laurillardi (Lund, 1842) (Casali \& Perini, 2016), Glossotherium robustum (Owen, 1842) (Pérez et al., 2010), Glyptodon clavipes Owen, 1839 (Burmeister, 1864, 1874), G. elongatus Burmeister, 1866 (Burmeister, 1871, 1874; Casali \& Perini, 2016), G. reticulatus Owen, 1845 (Burmeister, 1871, 1874), Lestodon armatus Gervais, 1855 (Tambusso et al., 2015), Megalonyx jeffersonii Desmarest, 1822 (Leidy, 1855; McDonald, 1977), Megatherium americanum Cuvier, 1796 (Owen, 1856; Pérez et al., 2010), Mionophropus cartellei De Iuliis, Gaudin \& Vicars, 2011 (De Iuliis et al., 2011), Mylodon darwinii Owen, 1839 (Allen, 1913), Nothrotheriops shastensis (Sinclair, 1905) (McDonald, 1977), N. maquinense (Lund, 1839) (Cartelle \& Fonseca, 1983), Panochthus tuberculatus (Owen, 1845) (Burmeister, 1864, 1874), Paramylodon harlani (Owen, 1840) (Pérez et al., 2000), Proeutatus sp. (Scott, 1905; Pérez et al., 2010), Prozaedyus sp. (Pérez et al., 2010), Scelidotherium leptocephalum Owen, 1840 (Pérez et al., 2010), Thalassocnus natans Muizon \& McDonald, 1995 (McDonald \& Muizon, 2002), y Valgipes bucklandi (Lund, 1839) (Casali \& Perini, 2016)]. Por su parte, de las 31 especies vivientes, también en 20 de ellas se conoce la morfología de esta estructura [Bradypus torquatus Illiger, 1811 (Casali \& Perini, 2016), B. tridactylus Linneo, 1758 (Gasc, 1967), B.variegatus Shinz, 1825 (Gasc, 1967), B. infuscatus Wagler, 1831 (Naples, 1986), Cabassous unicinctus (Linneo, 1758) (Casali \& Perini, 2016), Chaetophractus vellerosus (Gray, 1865) (Pérez et al., 2010), Ch. villosus (Desmarest, 1804) (Burmeister, 1874), Chlamyphorus truncatus Harlan, 1825 (Gibb et al., 2015), Choloepus didactylus (Linneo, 1758) (Flower, 1885), Ch. hoffmanni Peters, 1858 (Naples, 1986), Cyclopes didactylus (Linneo, 1758) (Reiss, 1997), Dasypus novemcinctus (Linneo, 1758) (Pérez et al., 2010), D. septemcinctus Linneo, 1758 (Casali \& Perini, 2016), Euphractus sexcinctus (Linneo, 1758)
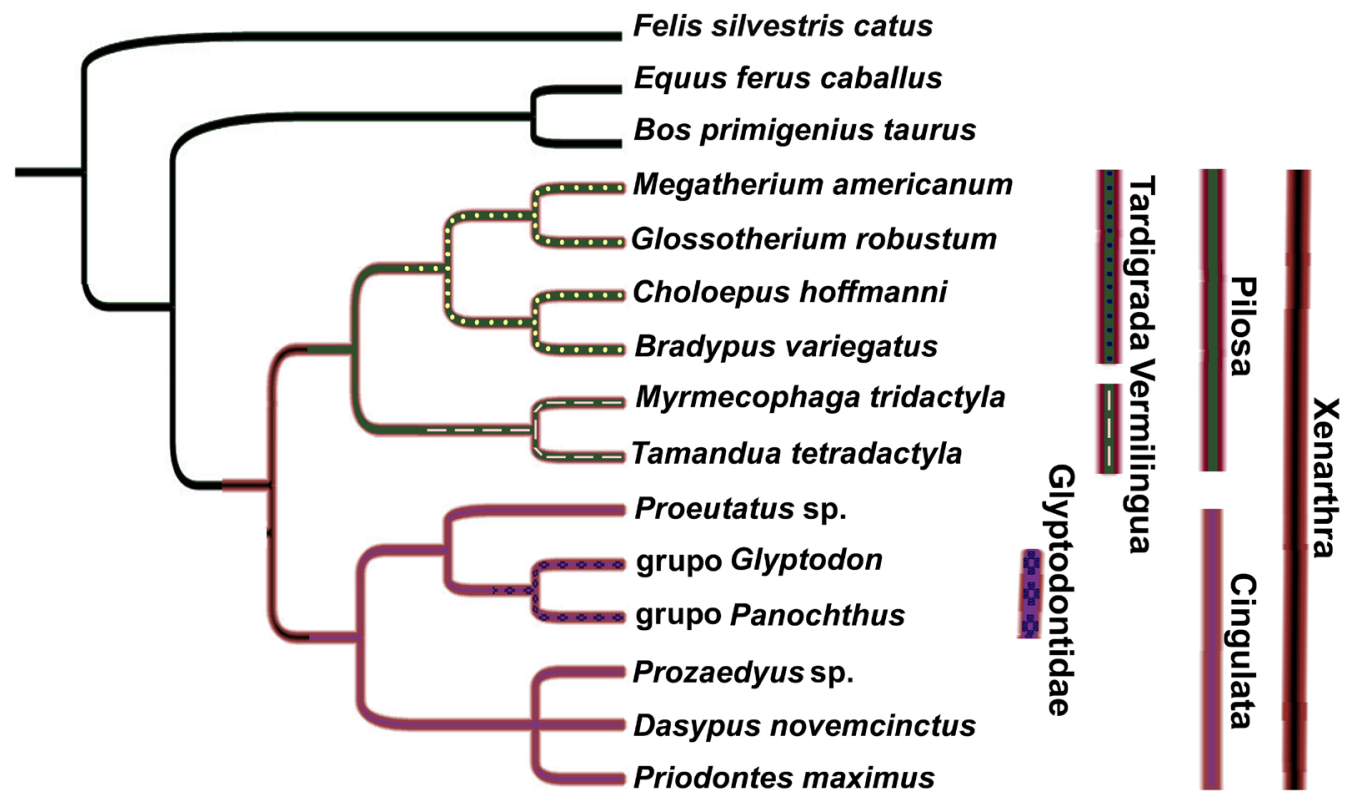

Figura 2. Árbol de máxima parsimonia con monofilias forzadas.

Figure 2. Most parsimonious tree with forced monophyly. 


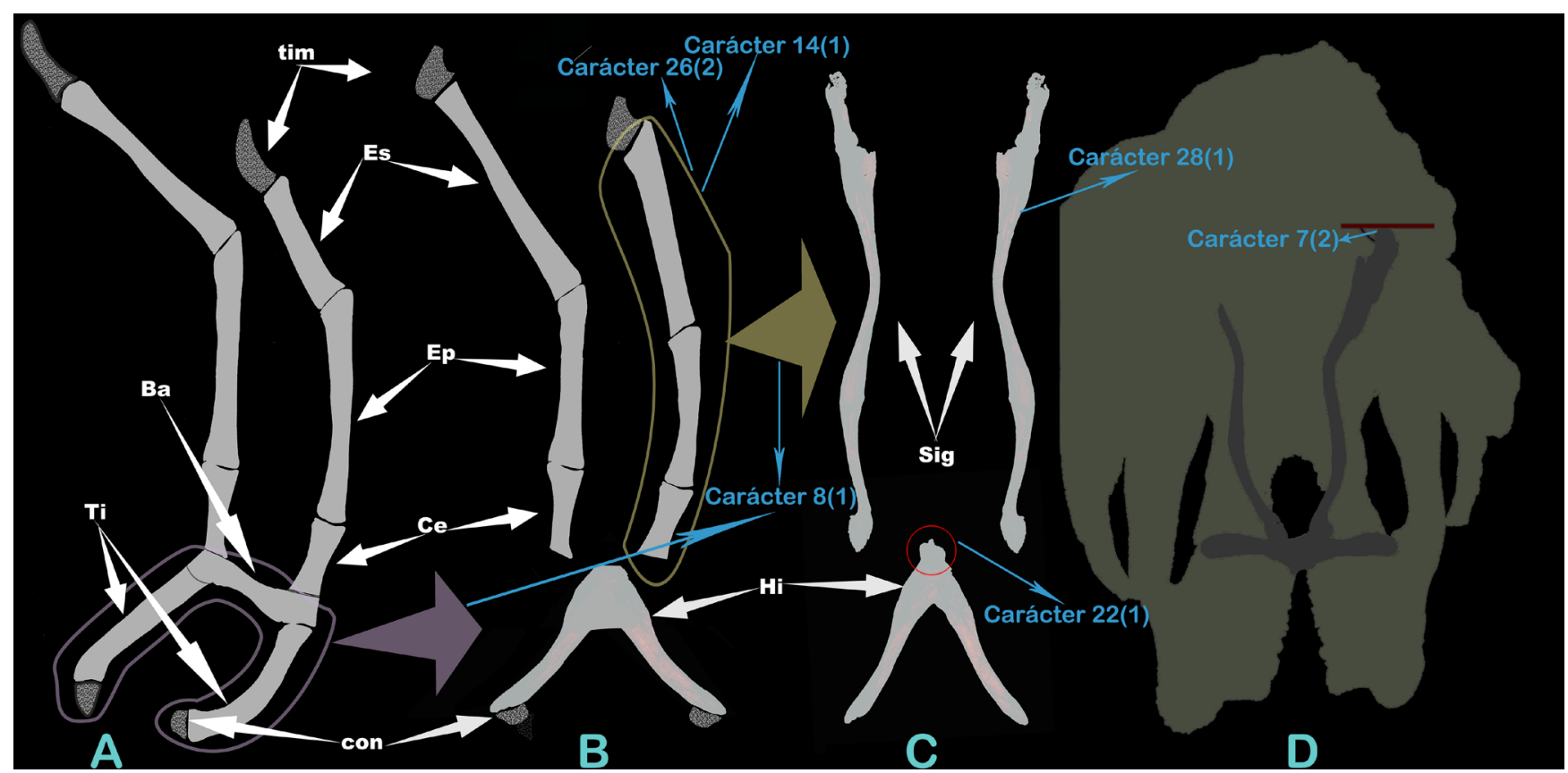

Figura 3. Sinapomorfías en gliptodóntidos. A-C, elementos óseos del aparato hioides. A, Felis silvestris catus (modificado de Jayne, 1898); B, Megatherium americanum (modificado de Pérez et al., 2010); C, Panochthus; D, vista posterior del cráneo. Carácter 7, ángulo entre el elemento que hace contacto con el cráneo y la base del proceso mastoideo (2) Mayor a $80^{\circ}$. Carácter 8, todos los elementos están fusionados (1) Sí. Carácter 14, estilohioides, epihioides y ceratohioides fusionados (1) Sí. Carácter 22, presencia de proceso lingual del hueso impar (1) presente. Carácter 26, máxima cantidad de elementos fusionados para formar un elemento (2) 3. Carácter 28, presencia de un único hueso contactando el cráneo con el hueso impar (1) Sí. Abreviaturas: Ba, basihioides; Ce, ceratohioides; con, condrohioides; Ep, epihioides; Es, estilohioides; Hi, hueso impar; Sig, sigmohioides; Ti, tirohioides; tím, tímpanohioides.

Figure 3. Synapomorphies in glyptodontids. A-C, bony elements of the hyoid apparatus. A, Felis silvestris catus (redrawn after Jayne, 1898); B, Megatherium americanum (redrawn after Pérez et al., 2010); C, Panochthus; D, posterior view of skull. Character 7, angle between the element that contact with the skull and the base of the mastoid process (2) Greater than $80^{\circ}$. Character 8, all elements are fused (1) Yes. Character 14, stylohyal, epihyal and ceratohyal fused (1) Yes. Character 22, presence of lingual process of the V-bone (1) present. Character 26, maximum amount of fused elements to form an element (2) 3. Character 28, presence of a single bone contacting the skull with the skull with the V-bone (1) Yes. Abbreviations: Ba, basihyal; Ce, ceratohyals; con, chondrohyals; Ep, epihyals; Es, stylohyals; Hi, V-bone; Sig, sigmohyals; Ti, thyrohyals; tim, tympanoyal.

(Casali \& Perini, 2016), Myrmecophaga tridactyla Linneo, 1758 (Naples, 1999), Priodontes maximus (Kerr, 1792) (Pérez et al., 2000), Tamandua mexicana (Saussure, 1860) (Reiss, 1997), T. tetradactyla Linneo (Casali \& Perini, 2016), Tolypeutes matacus (Desmarest, 1804) (Murie, 1874), y Tolypeutes tricinctus (Linneo, 1758) (Casali \& Perini, 2016)].

Desde una perspectiva filogenética, Pérez et al. (2010) mencionan que probablemente la fusión entre los tirohioides y el basihiodes, un ormando el hueso impar, sea una sinapomorfía de los xenartros; sin embargo, los autores aclaran que esta fusión también aparece en otros mamíferos, por ejemplo en el cetáceo Mesoplodon Gervais, 1850, en el lagomórfo Lepus Linneo, 1758 y en algunos suidos y primates antropoides. Siguiendo esta última idea, Wible (2010) demostró que en el grupo compuesto por los miembros de las familias Eriniceidae, Solenodentidae, Talpidae y Soricidae denominado Eulipotyphla -, dicha fusión es variable. Esto implica que en algunas especies está fusión aparece y en otra no. Sprague (1944) también había mencionado, además de las anteriormente citadas, a la familia Tenrecidae como poseedora de esta característica. Pérez et al. (2010), debido a la esporádica ocurrencia de dicha fusión en taxones que no están emparentados filogenéticamente, sugieren que esta característica es una adquisición independiente. En los análisis filogenéticos aquí presentados la mencionada característica aparece como una sinapomorfía de los xenartros, entre otras (ver Tabla 1). Los taxones que disponen de esta peculiar fusión difieren entre si notablemente en las características antes mencionadas; por ende, en los mamíferos que presentan los tirohioides y el basihiodes fusionados, no es posible dar una explicación a la presencia de dicha fusión.

Comparando el primer análisis elaborado en esta contribución con las filogenias tradicionales (vide supra) se conservan dos grupos naturales, xenartros y gliptodóntidos (ver Figura 1), soportados estos por sendas sinapomorfías (ver Tabla 1). No obstante, los resultados obtenidos en este análisis demuestran que los caracteres basados solo en los elementos óseos del aparato hioides no sirven para definir a los grandes grupos de xenartros, contradiciendo en esta idea a Casali \& Perini (2016). Estos autores realizaron una filogenia basada sobre 10 caracteres y 39 taxones, en la que dividen los xenartros en los grandes grupos tradicionales. En los 30 caracteres que se han utilizado para elaborar el presente análisis se ven reflejados todos los caracteres empleados por Casali \& Perini (2016), exceptuando el carácter 3, por los motivos expuestos más abajo. Sin embargo, en este análisis filogenético no se concuerda con las sinapomorfías que según Casali \& Perini (2016) sustentan estos grandes 
grupos. Las razones son: - en Pilosa - porque en el presente análisis se agregaron nuevos grupos externos y ya no existe dicha sinapomorfía (en este caso los ungulados) [carácter 9(1)]; - en Vermilingua - por tener en cuenta elementos, en el enunciado del carácter, que no están presentes en todos los xenartros [carácter 3(0)]; - en Tardigrada - porque en el presente análisis se agregaron nuevos grupos externos y ya no existe dicha sinapomorfía (en este caso los ungulados) [carácter 1(2)]; - en Cingulata - por tener en cuenta elementos, en el enunciado del carácter, que no están presentes en todos los xenartros [carácter 1(0), carácter 3(1)]; porque en el presente análisis se agregaron nuevos grupos externos y ya no existe dicha sinapomorfía (en este caso Felis silvestris catus) [carácter 2(1)]; por no coincidir con la codificación de los estados de carácter [carácter 8(1)], este último estado de carácter señalaría la presencia de una apófisis caudal en el basihiodes, el estado correcto para este carácter en los gliptodóntidos debería ser (0), ausente, ya que no poseen dicha apófisis, como se aprecia en la matriz y la fig. 2 , de Casali \& Perini (2016), pero también como indica la misma publicación, en dasipódidos está presente (Casali \& Perini 2016: matriz, fig. 1).
Producto del segundo análisis se aprecia un árbol filogenético con los grandes grupos en los que tradicionalmente se divide a los Xenarthra - Pilosa, Vermilingua, Tardigrada, Cingulata - (ver Figura 2), la cual se logra forzando tres monofilias - Vermilingua, Tardigrada, Cingulata -. Dicha clasificación tradicional se ve reflejada en diversos análisis filogenéticos (Gaudin \& Branham, 1998; Delsuc et al., 2001, 2002, 2003, 2012, 2016; Gaudin, 2003, 2004; Gaudin \& Wible, 2006; Möller-Krull et al., 2007; Gibb et al., 2015; Casali \& Perini, 2016). De los cuatro grupos de xenartros formados en este análisis solamente dos se ven soportados por sinapomorfías, los Vermilingua, por dos sinapomorfías y los Cingulata, por una sinapomorfía (ver Tabla 1); en tanto que, los otros dos grupos (Pilosa y Tardigrada) no están sustentados por sinapomorfías.

Mientras se testeaban las monofilias, un resultado interesante se dio cuando se llevó a cabo un análisis sin forzar la monofilia de los Pilosa (forzando la de Cingulata, Vermilingua y Tardigrada). En el árbol consecuente se manifiesta una vinculación filogenética más estrecha entre los Vermilingua y los Cingulata (Figura 4). Scillato-Yané \& Pascual (1985), basándose principalmente en caracteres

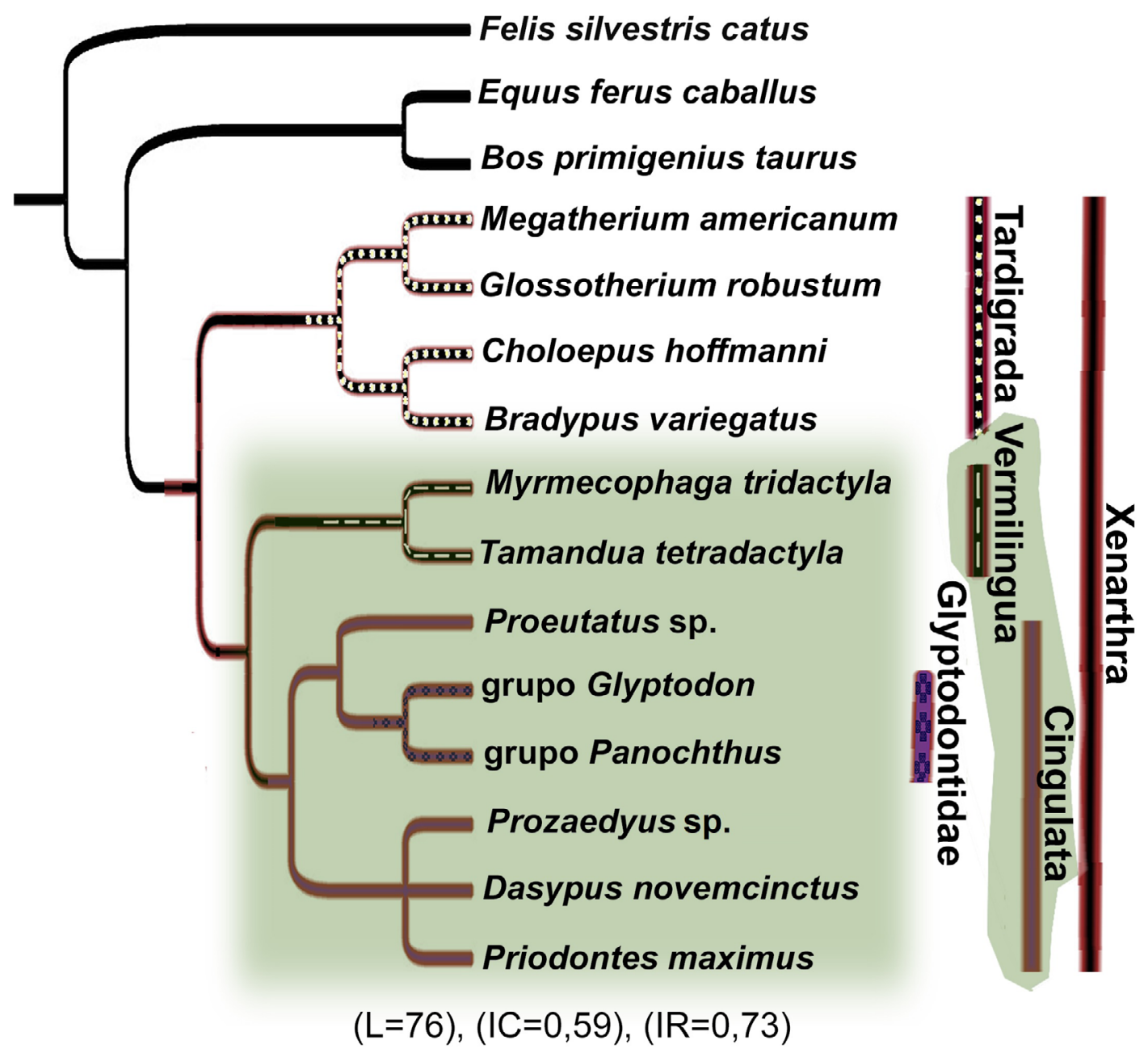

Figura 4. Árbol de máxima parsimonia con monofilias forzadas.

Figure 4. Most parsimonious tree with forced monophyly. 
dentarios, también vinculan más cercanamente a los Vermilingua y a los Cingulata, a diferencia de la agrupación clásica (ver Gray, 1869).

Pérez et al. (2010) describen y figuran el aparato hioides de Proeutatus sp., el cual consiste en un elemento alargado, que aparece articulado a la base del cráneo, y a este elemento lo denominan sigmohioides. Al discutir las implicancias filogenéticas, Pérez et al. (2010) sugieren que la presencia del sigmohioides podría ser una característica exclusiva que relacione estrechamente a eutatinos y gliptodóntidos (Engelmann, 1985; Vizcaíno \& Bargo, 1998; Gaudin \& Wible, 2006). Recientemente, Zamorano et al. (2018) demostraron que el sigmohioides de gliptodóntidos está formado por tres elementos: estilohioides-epihioides-ceratohioides; en tanto que el del género Proeutatus tiene solo dos elementos: estilohioides-epihioides (Scott, 1905; Pérez et al., 2010). No obstante, en los análisis filogenéticos de este trabajo Proeutatus sp. es el grupo hermano de gliptodóntidos.

En el contexto del primer análisis, interpretando los valores del soporte de Bremer (Goloboff \& Farris, 2001) y de Bootstrap (Soltis \& Soltis, 2003), puede inferirse que el clado de los gliptodóntidos es el que tiene mejor soporte. Este es, el único clado de xenartros que, en todos los análisis de esta contribución, representa un grupo con categoría taxonómica, familia Glyptodontidae (constituido en estos análisis por el grupo Glyptodon y el grupo Panochthus). Asimismo, son siempre el clado dentro de los xenartros sustentado por más sinapomorfías. En este contexto, es evidente el alto grado de especialización que posee el aparato hioides en esta peculiar grupo, así como su clara diferenciación con el resto de los taxones utilizados en los análisis. La estructura del aparato hioides en los gliptodóntidos se ve afectada junto con el resto del cráneo por el proceso de telescopización en el que el aparato masticatorio se desplaza debajo del neurocráneo (Fariña \& Parietti, 1983; Fariña \& Vizcaíno, 2001), como lo observaron Pérez et al. (2000, 2010) y Zamorano et al. (2018). Por la fusión de los elementos óseos del aparato hioides, en gliptodóntidos, solo hay tres huesos, los sigmohioides y el hueso impar. La conformación fusionada del sigmohioides descarta totalmente los ángulos entre los elementos, estilohioides-epihioides y epihioides-ceratohioides. A su vez, el sigmohioides tiene una disposición casi perpendicular a la del hueso impar. Este último se balancea en una sola dirección en sentido antero-posterior.

\section{CONCLUSIONES}

Los resultados del primer análisis demuestran que los caracteres basados solo en los elementos óseos del aparato hioides no sirven para definir los grandes grupos de xenartros. En un segundo análisis, en el que se forzaron monofilias para formar así las agrupaciones tradicionales en las que se dividen los xenartros, se observa que dos de ellas (Cingulata y Vermilingua) son sustentadas por una y dos sinapomorfías, respectivamente; en tanto que, los otros dos grupos (Pilosa y Tardigrada) no están soportados por ninguna sinapomorfía. Por otra parte, el grupo Glyptodon y el grupo Panochthus se recuperan siempre como grupos hermanos (Glyptodontidae es el único grupo con categoría taxonómica presente en estos análisis) y con Proeutatus sp. como el taxón más próximo. Los gliptodóntidos conforman el grupo de xenartros con vinculación filogenética más estrecha y con mayor fiabilidad.

\section{AGRADECIMIENTOS}

A J.N. Gelfo por los consejos y la colaboración con los análisis filogenéticos. A G. Gasparini por la colaboración con el resumo. A L. Soibelzon por la sugerencia de preparar el manuscrito. A N. Illarreguy, por la colaboración con el Abstract. A los revisores A. Zurita y D. Perea, por enriquecer el trabajo.

\section{REFERENCIAS}

Allen, G.M. 1913. A new Mylodon. Memoirs of the Museum of Comparative Zoology at Harvard College, 40:319-346.

Ameghino, F. 1889. Contribución al conocimiento de los mamíferos fósiles de la República Argentina. Actas de la Academia Nacional de Ciencias en Córdoba, 6:1-1027.

Ameghino, F. 1906. Les formations sedimentaries du Cretacé Superieur et du Tertiaire de Patagonie avec parallele entre leurs faunes mammalogiques et celles de l'ancien continents. Anales de Museo Nacional de Buenos Aires, 3:1-568.

Buckley, M.; Farina, R.A.; Lawless, C.; Tambusso, P.S.; Varela, L.; Carlini, A.A.; Powell, J.E. \& Martinez, J.G. 2015. Collagen sequence analysis of the extinct giant ground sloths Lestodon and Megatherium. PLoS ONE, 10:e139611. doi:10.1371/ journal.pone.0139611

Burmeister, H. 1864. Los objetos de la historia natural nuevos o poco conocidos conservados en este establecimiento. Anales del Museo Público de Buenos Aires, 1:121-465.

Burmeister, H. 1871. Osteologische Notizen zur Kunde der Panzerthiere Süd-Americas.A Das. Zungenbein der Edentata Loricata. Archiv für Anatomie, Physiologie und Wissenschaftliche Medicin, 21:418-429.

Burmeister, H. 1874. Monografía de los glyptodontes en el Museo Público de Buenos Aires. Anales del Museo Público de Buenos Aires, 2:355-412.

Cartelle, C. \& Fonseca, J.S. 1983.Contribuicao ao melhor conhecimento da pequena preguica terrícola Nothrotherium maquinense (Lund) Lydekker (1889). Lundiana, 2:127-181.

Casali, D.M. \& Perini, F.A. 2016. The evolution of hyoid apparatus in Xenarthra (Mammalia: Eutheria). Historical Biology, 6:777-788. doi:10.1080/08912963.2016.1241248

Cione, A.L.; Tonni, E.P.; Bond, M.; Carlini, A.A.; Pardiñas, U.F.J.; Scillato Yané, G.J.; Verzi, D. \& Vucetich, M.G. 1999. Ocurrence charts of Pleistocene mammals in the Pampean area, eastern Argentina. Quaternary of South America and Antarctic Peninsula, 12:53-73.

De Iuliis, G.; Gaudin, T.J. \& Vicars, M.J. 2011.A new genus and species of nothrotheriid sloth (Xenarthra, Tardigrada, Nothrotheriidae) from the late Miocene (Huayquerian) of Peru. Palaeontology, 54:171-205. doi:10.1111/j.14754983.2010.01001.x

de Jong, W.W.; Zweers, A.; Joysey, K.A.; Gleaves, J.T. \& Boulter, D. 1985. Protein sequence analysis applied to Xenarthran and Pholidote phylogeny. In: G.G. Montgomery (ed.) The evolution and ecology of armadillos, sloths, and vermilinguas, Smithsonian Institution Press, p. 65-76. 
Delsuc, F.; Catzeflis, F.M.; Stanhope, M.J. \& Douzery, E.J.P. 2001. The evolution of armadillos, anteaters and sloths depicted by nuclear and mitochondrial phylogenies: implications for the status of the enigmatic fossil Eurotamandua. Proceedings of the Royal Society of London, Biological Sciences, 268:1605-1615. doi:10.1098/rspb.2001.1702

Delsuc, F.; Gibb, G.C.; Kuch, M.; Billet, G.; Hautier, L.; Southon, J.; Rouillard, J.; Fernicola, J.C.; Vizcaíno, S.F. \& MacPhee, R.D.E. 2016. The phylogenetic affinities of the extinct glyptodonts. Current Biology, 26:R141-R156. doi:10.1016/j.cub.2016.01.039

Delsuc, F.; Scally, M.; Madsen, O.; Stanhope, M.J.; de Jong, W.W.; Catzeflis, F.M.; Springer, M.S. \& Douzery, E.J.P. 2002. Molecular phylogeny of the living xenarthrans and the impact of character and taxon sampling on the placental tree rooting. Molecular Biology and Evolution, 19:1656-1671. doi:10.1093/ oxfordjournals.molbev.a003989

Delsuc, F.; Stanhope, M.J. \& Douzery, E.J.P. 2003. Molecular systematic of armadillos (Xenarthra, Dasypodidae): contribution of maximum likelihood and Bayesian analyses of the mitochondrial and nuclear genes. Molecular Phylogenetics and Evolution, 28:261-275. doi:10.1016/S1055-7903(03)00111-8

Delsuc, F.; Superina, M.; Tilak, M.K; Douzery, E.J.P. \& Hassanin, A. 2012. Molecular phylogenetics unveils the ancient evolutionary origins of the enigmatic fairy armadillos. Molecular Phylogenetics and Evolution, 62:673-680. doi:10.1016/j. ympev.2011.11.008

Engelmann, G.F. 1985. The phylogeny of the Xenarthra. In: G.G. Montgomery (ed.) The evolution and ecology of armadillos, sloths, and vermilinguas, Smithsonian Institution Press, p. 51-64.

Fariña, R.A. 2001. Física y Matemáticas para reconstruir la vida en el pasado. Actas de Fisiología, 6:45-70.

Fariña, R.A. \& Parietti, M. 1983.Uso del método RFTRA en la comparación de la morfología craneana en Edentata. In: JORNADAS DE CIENCIAS NATURALES, 3, 1983. Resúmenes y Comunicaciones, Montevideo, p. 106-108.

Fariña, R.A. \& Vizcaíno, S.F. 2001. Carved teeth and strange jaws: how glyptodonts masticated. Acta Paleontologica Polonica, 46:87-102.

Flower, W.H. 1885. An introduction to the osteology of the Mammalia. London, Macmillan, 482 p.

Gasc, J.P. 1967. Squelette hyobrachial. In: P.P. Grassé (ed.) Traité de zoologie, anatomie, systématique, biologie, Masson et Cie, p. 550-583.

Gaudin, T.J. 1995. The ear region of edentates and the phylogeny of the Tardigrada (Mammalia, Xenarthra). Journal of Vertebrate Paleontology, 15:672-705. doi:10.1080/02724634.1995.100 11255

Gaudin, T.J. 1999. The morphology of xenarthrous vertebrae (Mammalia, Xenarthra). Fieldiana, 41:1-38.

Gaudin, T.J. 2004. Phylogenetic relationships among sloths (Mammalia, Xenarthra, Tardigrada): the craniodental evidence. Zoological Journal of the Linnean Society, 140:255-305. doi:10.1111/j.1096-3642.2003.00100.x

Gaudin, T.J. \& Branham, D.G. 1998. The phylogeny of the Myrmecophagidae (Mammalia, Xenarthra, Vermilingua) and the relationship of Eurotamandua to the Vermilingua. Journal of Mammalian Evolution, 32:237-265. doi:10.1023/A:1020512529767

Gaudin, T.J. \& Croft, D.A. 2015. Paleogene Xenarthra and the evolution of South American mammals. Journal of Mammalogy, 96:622-634. doi:10.1093/jmammal/gyv073

Gaudin, T.J. \& McDonald, H. G. 2008. Morphology-based investigations of the phylogenetic relationships among extant and fossil xenarthrans. In: S.F. Vizcaíno \& W.J. Loughry (eds.) The biology of the Xenarthra, University Press of Florida, p. 24-36.

Gaudin, T.J. \& Wible, J.R. 2006. The phylogeny of living and extinct armadillos (Mammalia, Xenarthra, Cingulata): a craniodental analysis. In: M.T. Carrano; T.J. Gaudin; R.W. Blob \& J.R. Wible (eds.) Amniote Paleobiology: perspectives on the evolution of mammals, birds and reptiles, University of Chicago Press, p. 153-198.

Gaudin, T.J.; Wible, J.R.; Hopson, J.A. \& Turnbull, W.D. 1996. Reexamination of the morphological evidence for the Cohort Epitheria (Mammalia, Eutheria). Journal of Mammalian Evolution, 3:31-79. doi:10.1007/BF01454253

Gibb, G.C.; Condamine, F.L.; Kuch, M.; Enk, J.; Moraes-Barros, N.; Superina, M.; Poinar, H.N. \& Delsuc, F. 2015. Shotgun mitogenomics provides a reference phylogenetic framework and timescale for living Xenarthrans. Molecular Biology and Evolution, 33:621-642. doi:10.1093/molbev/msv250

Goloboff, P.A. \& Farris, J.S. 2001. Methods for quick consensus estimation. Cladistics, 17:S26-S34. doi:10.1006/clad.2000.0156

Goloboff, P.A.; Farris, J. \& Nixon, K. 2008a. TNT, a free program for phylogenetic analysis. Cladistics, 24:774-786. doi:10.1111/ j.1096-0031.2008.00217.x

Goloboff, P.A.; Farris, J. \& Nixon, K. 2008b. TNT: tree analysis using new technology, version 1.1. Available at http://www.zmuc.dk/ public/phylogeny/tnt; accessed on 04/12/2019.

Gray, J.E. 1869. Catalogue of carnivorous, pachydermatous and edentate Mammalia in the British Museum. London, British Museum, 398 p.

Hoffstetter, R. 1958. Xenarthra. In: J. Piveteau (ed.) Traité de Paléontologie, J.-B. Baillière, p. 535-636.

Hoffstetter, R. 1982. Les edentés xenarthres, un groupe singulier de la faune néotropicale (origine, affinités, radiation adaptative, migrations et extinctions). In: E.M. Galitelli (ed.) Palaeontology, essential of historical geology, S.T.E.M. Mucchi, p. 383-443.

Jayne, H. 1898. Mammalian anatomy. The American Journal of the Medical Sciences, 116:210-227.

König, H.E. \& Liebich, H.G. 2005. Anatomía de los animales domésticos: texto y atlas en color.Tomo 1, Aparato locomotor. Buenos Aires, Editorial Médica Panamericana, 288 p.

Kraglievich, J.L. 1934. La antigüedad de las faunas de Monte Hermoso y Chapadmalal, deducidas de su comparación con las que le precedieron y sucedieron. Montevideo, Imprenta "El Siglo Ilustrado", $136 \mathrm{p}$.

Leidy, J. 1855. A memoir on the extinct sloth tribe of North America. Smithsonian Contributions to Knowledge, 7:1-68.

Maddison, W.P. \& Maddison, D.R. 2016. Mesquite: a modular system for evolutionary analysis (ver. 3.10). Available at www. mesquiteproject.org; accessed on 04/12/2019.

Madsen, O.; Scally, M.; Douady, C.J.; Kao, D.J.; Debry, R.W.; Adkins, R.; Amrine, H.M.; Stanhope, M.J.; De Jong, W.W. \& Springer, M.S. 2001. Parallel adaptative radiations in two major clades of placental mammals. Nature, 409:610-614. doi: $10.1038 / 35054544$

McDonald, H.G. 1977. Description of the osteology of the exctinct gravigrade edentate Megalonyx with observations on its ontogeny, phylogeny and functional anatomy. University of Florida, Master's thesis, $140 \mathrm{p}$.

McDonald, H.G. 2003. Xenarthran skeletal anatomy: primitive or derived (Mammalia, Xenarthra). Senckenbergiana Biologica, 83:5-17.

McDonald, H.G. 2005. Paleoecology of extinct xenarthrans and the Great American Biotic Interchange. Bulletin of the Florida Museum of Natural History, 45:313-333. 
McDonald, H.G. \& de Muizon, C. 2002. The cranial anatomy of Thalassocnus (Xenarthra, Mammalia), a derived Nothrothre from the Neogene of the Pisco Formation (Peru). Journal of Vertebrate Paleontology, 22:349-365. doi:10.1671/02724634 (2002)022[0349:TCAOTX]2.0.CO;2

McKenna, M.C. \& Bell, S.K. 1997. Classification of Mammals above the species level. New York, Columbia University Press, 640 p.

Moller-Krull, M.; Delsuc, F.; Churakov, G.; Marker, C.; Superina, M.; Brosius, J.; Douzery, E.J.P. \& Schmitz, J. 2007. Retroposed elements and their flanking regions resolve the evolutionary history of xenarthran mammals (Armadillos, anteaters, and sloths). Molecular Biology and Evolution, 24:2573-2582. doi:10.1093/molbev/msm201

Mones, A. 1986. Palaeovertebrata Sudamericana. Catálogo sistemático de los vertebrados fósiles de América del Sur. Parte I. Lista preliminar y bibliografía. Courier Forschungsinstitut Senckenberg, 82:1-625.

Murie, D.J. 1874. On the habits, structure, and relations of the three-banded armadillo (Tolypeutes conurus, Is. Geoff.). Transactions of the Linnean Society of London, 30:71-132. doi:10.1111/j.1096-3642.1874.tb00003.x

Murphy, W.J.; Eizirik, E.; Johnson, W.E.; Zhang, Y.P.; Ryder, O.A. \& O'Brien, S.J. 2001. Molecular phylogenetics and the origins of placental mammals. Nature, 409:614-618. doi: $10.1038 / 35054550$

Naples, V.L. 1986. The morphology and function of the hyoid region in the tree sloths, Bradypus and Choloepus. Journal of Mammalogy, 67:712-724. doi:10.2307/1381132

Naples, V.L. 1999. Morphology, evolution and function of feeding in the giant anteater (Myrmecophaga tridactyla). Journal of Zoology, 249:19-41. doi:10.1111/j.1469-7998.1999.tb01057.x

O’Leary, M.A.; Bloch, J.I.; Flynn, J.J.; Gaudin, T.J.; Giallombardo, A.; Giannini, N.P.; Goldberg, S.L.; Kraatz, B.P.; Luo, Z.X. \& Meng J. 2013. The placental mammal ancestor and the Post-K$\mathrm{Pg}$ radiation of placentals. Science, 339:662-667. doi:10.1126/ science. 1229237

Owen, R. 1856. On the Megatherium (Megatherium americanum, Cuvier and Blumenbach). Part III. The skull. Philosophical Transactions of the Royal Society, 146:571-589. doi:10.1098/ rstl.1856.0028

Paula Couto, J.C. 1957. Sôbre um gliptodonte do Brasil. Rio de Janeiro, Departamento Nacional de Produção Mineral, Divisão de Geologia e Mineralogia, 37 p. (Boletim 165).

Pérez, L.M.; Scillato-Yané, G.J. \& Vizcaíno, S.F. 2000. Estudio morfofuncional del aparato hiodeo de Glyptodon cf. clavipes (Cingulata: Glyptodontidae). Ameghiniana, 37:293-299.

Pérez, L.M.; Toledo, N.; De Iuliis, G.; Bargo, M.S. \& Vizcaíno, S.F. 2010 .Morphology and function of the hyoid apparatus of fossil xenarthrans (mammalia). Journal of Morphology, 271:1119-1133. doi:10.1002/jmor.10859

Porpino, K.O.; Fernícola, J.C. \& Bergqvist, L.P. 2009. A new cingulate (Mammalia: Xenarthra) Pacharmatherium brasiliensis sp. nov., from the late Pleistocene of the Northeastern Brazil. Journal of Vertebrate Paleontology, 29:881-893. doi:10.1671/039.029.0305

Redford, K.H. \& Eisenberg, J.F. 1992. Mammals of the Neotropics. The Southern Cone. Chicago, University of Chicago Press, $430 \mathrm{p}$.

Reiss, K.Z. 1997. Myology of the feeding apparatus of myrmecophagid anteaters (Xenarthra: Myrmecophagidae). Journal of Mammalian Evolution, 4:87-117. doi:10.1023/A:1027366129277

Rose, K.D.; Emry, R.J.; Gaudin, T.J. \& Storch, G. 2005. Xenarthra and Pholidota. In: K.D. Rose \& J.D. Archibald (eds.) The rise of placental mammals: origins and relationships of the major extant clades, Johns Hopkins University Press, p. 106-126.

Rusconi, C. 1937. Contribución al conocimiento de la geología de la ciudad de Buenos Aires y sus alrededores y referencia de su fauna. Actas de la Academia Nacional de Ciencias de Córdoba, 10:177-294.

Scillato-Yané, G.J. 1976. Sobre un Dasypodidae (Mammalia, Xenarthra) de Edad Riochiquense (Paleoceno Superior) de Itaboraí, Brasil. Anais da Academia Brasileira de Ciências, 48:529-530.

Scillato-Yané, G.J. 1982. Los Dasypodidae (Mammalia, Edentata) del Plioceno y Pleistoceno de Argentina. Universidad Nacionalde La Plata, Tesis doctoral, $244 \mathrm{p}$.

Scillato-Yané, G.J. 1986. Los Xenarthra fósiles de Argentina (Mammalia, Edentata). In: CONGRESO ARGENTINO PALEONTOLOGÍA Y BIOESTRATIGRAFÍA, 5, 1986. Actas, Mendoza, p. 151-155.

Scillato-Yané, G.J. \& Carlini, A.A. 1995. Estado actual del estudio de los Xenarthra. In: SIMPOSIO ESTADO ACTUAL DEL ESTUDIO DE LOS XENARTHRA, 1, 1995. Resúmenes, La Plata, p. 27.

Scillato-Yané, G.J. \& Carlini, A.A. 1998. Un Gigantesco Gliptodonte en los alrededores de la Ciudad de La Plata. Revista Museo de La Plata, 11:45-48.

Scillato-Yané, G.J.; Carlini, A.A.; Vizcaíno, S.F. \& Ortiz-Jaureguizar, E. 1995. Xenarthra. In: M.T. Alberdi; E.P. Tonni \& G. Leone (eds) Evolución biológica y climática de la región Pampeana durante los últimos cinco millones de años. Un ensayo de correlación con el Mediterraneo Occidental, CSIC, p. 183-209.

Scillato-Yané, G.J. \& Pascual, R. 1985. Un peculiar Xenarthra del Paleoceno medio de Patagonia (Argentina). Su importancia en la sistemática de los Paratheria. Ameghiniana, 21:173-176.

Scott, W.B. 1905. Mammalia of the Santa Cruz beds.Paleontology II. Part I Edentata II Insectivora III Glires. Princeton, Princeton University Expedition to Patagonia, 489 p. (Reports 5).

Shoshani, J. \& Marchant, G. 2001. Hyoid apparatus: a little known complex of bones and its "contribution" to proboscidean evolution. In: G. Cavarretta; P. Gioia; M. Mussi \& M. Palombo (eds.) The World of Elephants, p. 668-675.

Sisson, S. \& Grossman, J.D. 1977. Anatomía de los animales domésticos. Barcelona, Salvat Editores. 952 p.

Slater, G.; Cui, P.; Forasiepi, A.M.; Lenz, D.; Tsangaras, K.; Voirin, B.; de Moraes-Barros, N.; MacPhee, R.D.E. \& Greenwood, A.D. 2016. Evolutionary relationships among extinct and extant sloths: the evidence of mitogenomes and retroviruses. Genome Biology and Evolution, 8:607-621. doi:10.1093/gbelevw023

Soibelzon, L.H.; Zamorano, M.; Scillato-Yané, G.J.; Piazza, D.; Rodríguez, S.; Soibelzon, E.; Tonni, E.P.; San Cristóbal, J. \& Beilinson, E. 2012. Un glyptodontidae de gran tamaño en el Holoceno temprano de la región Pampeana. Revista Brasileira de Paleontologia, 15:113-122. doi:10.4072/rbp.2012.1.09

Soibenzon, E.; Zurita, A.E. \& Carlini, A.A. 2006. Glyptodon munizi (Mammalia, Cingulata, Glyptodontidae): redescripción y anatomía. Ameghiniana, 43:377-385.

Soltis, P.S. \& Soltis, D.E. 2003. Applying the bootstrap in phylogeny reconstruction. Statistical Science, 2:256-267. doi:10.1214/ ss/1063994980

Sprague, J.M. 1944. The hyoid region in the insectivora. American Journal of Anatomy, 74:175-216. doi:10.1002/aja.1000740203

Tambusso, P.S.; Mcdonald, H.G. \& Fariña R.A. 2015. Description of the stylohyal bone of a giant sloth (Lestodon armatus). Palaeontologia Electronica, 18.1.18A. doi:10.26879/506 
Taylor, W.T. \& Webber, J.T. 1951. Functional mammalian anatomy. New York, Litton Educational Publishing. 575 p.

Vizcaíno, S.F. \& Bargo, M.S. 1998.The masticatory apparatus of the armadillo Eutatus (Mammalia. Cingulata) and some allied genera: paleobiology and evolution. Paleobiology, 24:371-383. doi:10.1666/0094-8373(1998)024[0371:TMAOTA]2.3.CO;2

Vizcaíno, S.F.; Carlini, A.A. \& Scillato-Yané, G.J. 1990. Los Dasypodidae miocénicos (Mammalia, Xenarthra): implicancias en la distribución actual de la Familia. In: REUNIÓN CONJUNTA DE LA SOCIEDAD ARGENTINA PARA EL ESTUDIO DE LOS MAMÍFEROS (SAREM) Y DE LA AMERICAN SOCIETY OF MAMMALOGIST (AMS), 11, 1990. Actas, Buenos Aires, p. 60.

Vizcaíno, S.F. \& De Iuliis, G. 2003. Evidence for advanced carnivory in fossil armadillos (Mammalia: Xenarthra: Dasypodidae). Paleobiology, 29:123-138. doi:10.1666/00948373(2003)029\%3C0123:EFACIF\%3E2.0.CO;2

Vizcaíno, S.F.; Fariña, R.A.; Bargo, M.S. \& De Iuliis, E. 2004. Functional and phylogenetic assessment of the masticatory adaptations in Cingulata (Mammalia, Xenarthra). Ameghiniana, 41:651-664.

Wetzel, R.M. 1982. Systemathcs, distribution, ecology, and conservation of South American edentates. In: M.A. Mares \& H.H. Genoways (eds.) Mammalian biology in South America, University of Pitsburgh, p. 345-375

Wetzel, R.M. 1985. The identification and distribution of recent Xenarthra (=Edentata). In: G.G. Montgomery (ed.) Ecology of armadillos, sloths, and vermilinguas, Smithsonian Institution Press, p. 5-21.

Wible, J.R. 2010. On the hyoid and larynx of the Hispaniolan solenodon, Solenodon paradoxus Brandt, 1833 (Mammalia, Lipotyphla, Solenodontidae). Annals of Carnegie Museum, 79:29-38. doi:10.2992/007.079.0102
Zamorano, M. 2012. Los Panochthini (Xenarthra, Glyptodontidae): sistemática y evolución. Universidad Nacional de La Plata, Tesis Doctoral, $278 \mathrm{p}$.

Zamorano, M.; Scillato-Yané, G.J.; Soibelzon, E.; Soibelzon, L.H.; Bonini, R. \& Rodriguez, S. 2018. Hyoid apparatus of Panochthus sp. (Xenarthra; Glyptodontidae) from the Late Pleistocene of the Pampean region (Argentina). Comparative description and muscle reconstruction. Neues Jahrbuch für Geologie und Paläontologie, 288:205-219. doi:10.1127/njgpa/2018/0733

Zamorano, M.; Scillato-Yane, G.J. \& Zurita, A.E. 2014.Revisión del género Panochthus (Xenarthra, Glyptodontidae). Revista del Museo de La Plata, 14:1-46.

Zurita, A.E.; Miño-Boilini, A.R.; Soibelzon, E.; Carlini, A.A. \& Paredes-Ríos, F. 2009. The diversity of Glyptodontidae (Xenarthra, Cingulata) in the Tarija Valley (Bolivia): systematic, biostratigraphic and paleobiogeographic aspects of a particular assemblage. Neues Jahrbuch für Geologie und Paläontologie, 251:225-237. doi:10.1127/0077-7749/2009/0251-0225

Zurita, A.E.; Scillato-Yané, G.J.; Ciancio, M.; Zamorano, M. \& González Ruiz, L.R. 2016. Los Glyptodontidae (Mammalia, Xenarthra): historia biogeográfica y evolutiva de un grupo particular de mamíferos acorazados. In: F.L. Agnolin; G.L. Lio; F.B. Egli; N.R. Chimento \& F.E. Novas (eds.) Historia evolutiva y paleobiogeográfica de los vertebrados de América del Sur, MACN, p. 249-262.

Received in 17 November, 2018; accepted in 12 April, 2019. 
Apéndice 1. Bibliografía de la cual se extrajeron los datos morfológicos de los taxones utilizados en el análisis filogenético. Appendix 1. Bibliography which the morphological data of the taxa used in the phylogenetic analysis were extracted.

\begin{tabular}{|c|c|}
\hline Taxones & Datos bibliográficos \\
\hline Bos primigenius taurus & Pérez et al. (2000), König \& Liebich (2005) \\
\hline Bradypus variegatus & Gasc (1967), Naples (1986) \\
\hline Choloepus hoffmanni & Gasc (1967), Naples (1986) \\
\hline Dasypus novemcinctus & Gasc (1967), Pérez et al. (2010) \\
\hline Equus ferus caballus & Sisson \& Grossman (1977), König \& Liebich (2005) \\
\hline Felis silvestris catus & König \& Liebich (2005) \\
\hline Glossotherium robustum & Pérez et al. (2010) \\
\hline Megatherium americanum & Pérez et al. (2010) \\
\hline Myrmecophaga tridactyla & Reiss (1997), Naples (1999), Casali \& Perini (2016) \\
\hline Priodontes maximus (kerr) & Pérez et al. (2000) \\
\hline Proeutatus sp. & Scott (1905), Pérez et al. (2010) \\
\hline Prozaedyus sp. & Pérez et al. (2010) \\
\hline Tamandua tetradactyla & Naples (1999), Casali \& Perini (2016) \\
\hline \multicolumn{2}{|l|}{ "grupo Glyptodon" } \\
\hline Glyptodon cf. G. clavipes & Pérez et al. (2000); la presente publicación \\
\hline G. reticulatus & Burmeister $(1871,1874)$ \\
\hline G. clavipes & Burmeister $(1864,1874)$ \\
\hline G. elongatus & Burmeister $(1871,1874)$, Casali \& Perini (2016) \\
\hline \multicolumn{2}{|l|}{ "grupo Panochthus" } \\
\hline Panochthus sp. & La presente publicación \\
\hline P. tuberculatus (Owen) & Burmeister $(1864,1874)$ \\
\hline
\end{tabular}

Apéndice 2. Lista de caracteres y matriz (taxones/caracteres y sus estados de carácter). Appendix 2. List of characters and matrix (taxa/characters and their character states).

Carácter 1. Cantidad de elementos pares libres [nuevo]
0.4
1. 3
2. 1
3. 0

Carácter 2. Cantidad de elementos que componen el hueso impar [nuevo]

$$
\text { 0. } 1
$$

1. 2

Carácter 3. Articulaciones entre los elementos [nuevo]
0.4
1. 3
2. 2
3. 1

Carácter 4. Cantidad de elementos fusionados [nuevo]
0.1
1. 2
2. 4
3. 5

Carácter 5. Estilohioides fusionados [nuevo]

1. Sí 
Carácter 6. Epihioides fusionados [nuevo]

$0 . \quad \mathrm{No}$

1. Sí

Carácter 7. Ángulo entre el elemento que hace contacto con el cráneo y la base del proceso mastoideo de éste último [nuevo]

0 . Menor a $40^{\circ}$

1. Entre $40^{\circ}$ y $80^{\circ}$

2. Mayor a $80^{\circ}$

Carácter 8. Todos los elementos están fusionados [nuevo]

0 . No

1. Sí

Carácter 9. Tirohioides fusionados [nuevo]

$0 . \quad$ No

1. Sí

Carácter 10. Estilohioides y epihioides fusionados [modificado de Casali \& Perini, 2016, carácter 4]

$0 . \quad$ No

1. Sí

Carácter 11. Epihioides y ceratohioides fusionados [modificado de Casali \& Perini, 2016, carácter 6]

$0 . \quad$ No

1. Sí

Carácter 12. Forma del hueso impar [nuevo]

$0 . \quad-$

1. $\mathrm{V}$

Carácter 13. Basihioides y tirohioides fusionados [modificado de O’Leary et al. 2013, carácter 1258]

$0 . \quad$ No

1. Sí

Carácter 14. Estilohioides, epihioides y ceratohioides fusionados [nuevo ]

$0 . \quad$ No

1. Sí

Carácter 15. El elemento más largo está formado por huesos fusionados [nuevo]

$0 . \quad$ No

1. Sí

Carácter 16. Longitud del estilohioides en relación con la longitud del epihioides [modificado de Gaudin, 2004, carácter77]

$0 . \quad$ Aproximadamente igual

1. Mayor

2. Algunos de estos huesos está fusionado

Carácter 17. Tamaño comparado del epihioides y el ceratohioides [nuevo]

0. Epihioides menor que el ceratohiodes

1. Epihiodes mayor que el ceratohiodes

2. Algunos de los huesos está fusionado

Carácter 18. Proporción en que el estilohioides supera al ceratohioides [nuevo]

0. Menos del doble

1. Más del doble, pero menos de 7 veces

2. Más de 7 veces

3. Algunos de los huesos está fusionado

Carácter 19. Los elementos pares son predominantemente [nuevo ]

0. Rectos

1. Curvos

Carácter 20. El ancho máximo del elemento estilohioides (o sigmohioides) está en el extremo distal [nuevo]

0. Sí

1. No, se encuentra más proximalmente

Carácter 21. Presencia de ceratohioides [modificado de O’Leary et al., 2013, carácter 1260]

0 . Presente, como elemento independiente

1. Presente, como elemento fusionado

2. Ausente 
Carácter 22. Presencia de proceso lingual del hueso impar [modificado de O’Leary et al., 2013, carácter 1255]

$0 . \quad$ Ausente

1. Presente

Carácter 23. Presencia de la apófisis caudal del hueso impar (o basihioides) [modificado de Casali \& Perini, 2016, carácter 8]

0. Ausente

1. Presente

Carácter 24. Presencia del proceso transverso del extremo distal del hueso impar (o tirohioides) [modificado de O’Leary et al., 2013, carácter 1259]

0 . Ausente

1. Presente

Carácter 25. El tamaño del estilohioides es mayor al tamaño de todos los otros elementos pares juntos [nuevo]

0. Sí

1. No

2. Estilohioides fusionado

Carácter 26. Máxima cantidad de elementos fusionados para formar un elemento [nuevo]

$0 . \quad 1$, Solamente el basihioides

1. 2

2. 3

Carácter 27. Cantidad de huesos compuestos por elementos fusionados [nuevo]

0 . Solamente el basihioides

1. 1

2. 2

Carácter 28. Presencia de un único hueso contactando el cráneo con el hueso impar [nuevo]

0 . No

1. Sí

Carácter 29. El hueso fusionado es más grande que el resto de los elementos juntos [nuevo]

0 . No tiene huesos fusionados

1. No

2. Sí

Carácter 30. El extremo distal del estilohioides (o sigmohioides) en relación con al resto del hueso [modificado de Casali \& Perini, 2016, carácter 1]

0 . Más grande

1. Mantiene las proporciones

2. Más chico

\begin{tabular}{|c|c|c|c|c|c|c|c|c|c|c|c|c|c|c|c|c|c|c|c|c|c|c|c|c|c|c|c|c|c|c|}
\hline Car & 1 & 2 & 3 & 4 & 5 & 6 & 7 & 8 & 9 & 0 & 11 & 12 & 13 & 14 & 15 & 6 & 17 & $1 \xi$ & 19 & 20 & 21 & 22 & 23 & 24 & 25 & 26 & 2 & 28 & 29 & \\
\hline is $c a$ & 0 & 0 & 0 & 0 & 0 & 0 & & 0 & 0 & 0 & 0 & 0 & 0 & 0 & 0 & & 1 & 1 & 0 & 0 & & 0 & & 0 & & & 0 & & & \\
\hline Equi & 0 & 0 & 0 & 0 & 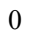 & 0 & & 0 & c & 0 & 0 & 0 & & 0 & 0 & ? & 2 & ? & 0 & 0 & & 1 & & 1 & & & 0 & & & \\
\hline Bes $n r$ & 0 & 0 & 0 & 0 & 0 & 0 & 1 & 0 & 0 & 0 & 0 & 0 & & & 0 & & 0 & & 0 & 0 & & 1 & & 1 & & & 0 & & & \\
\hline Myrme & 1 & 1 & 1 & 1 & 0 & 0 & 0 & 0 & 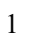 & 0 & 0 & 1 & 1 & 0 & 0 & 1 & 1 & 1 & 1 & 1 & 0 & 0 & & 0 & & & 1 & & & \\
\hline Tam & 2 & 1 & 2 & 2 & 0 & 1 & 0 & 0 & 1 & 0 & 1 & 1 & 1 & 0 & 0 & 2 & 2 & 2 & 1 & 1 & & 0 & 0 & 0 & & & 2 & & & \\
\hline & 1 & 1 & 1 & 1 & 0 & 0 & 1 & 0 & 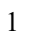 & 0 & 0 & 1 & 1 & O & 0 & & 1 & 1 & 0 & 0 & & 0 & & 0 & c & & & & & \\
\hline Glos & 1 & 1 & 1 & 1 & 0 & 0 & 1 & 0 & 1 & 0 & 0 & 1 & 1 & 0 & 0 & 1 & 1 & 1 & 0 & 0 & & 0 & & 0 & & & & & & \\
\hline Choloe & 2 & 2 & 2 & 2 & 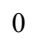 & 1 & 0 & 0 & 1 & 0 & 1 & 1 & 1 & 0 & 0 & ? & 2 & 2 & 1 & 0 & & 0 & & 0 & & & ? & & & \\
\hline $\mathrm{Bra}$ & 2 & 1 & 2 & 2 & 0 & 1 & 0 & 0 & 1 & 0 & 1 & 1 & 1 & 0 & 0 & & 2 & 2 & 1 & 0 & & 0 & ( & 1 & 1 & & & & & \\
\hline Das. & 1 & 1 & 1 & 1 & 0 & 0 & 0 & 0 & 1 & 0 & 0 & 1 & 1 & 0 & 0 & 0 & 1 & 0 & 0 & 1 & 0 & 0 & & 1 & 1 & & & 0 & & \\
\hline Priodont & 1 & 1 & 1 & 1 & 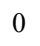 & 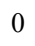 & 0 & 0 & 1 & 0 & 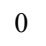 & 1 & 1 & 0 & . & 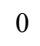 & 1 & 0 & . & 1 & 0 & 0 & 1 & 1 & 1 & & 1 & & & \\
\hline Pro & ? & 1 & 1 & $?$ & 0 & 0 & 0 & 0 & 1 & 0 & 0 & 1 & 1 & 0 & 0 & 0 & $?$ & $?$ & 0 & 1 & ? & 0 & ? & 1 & 1 & 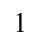 & 1 & & 0 & \\
\hline Pro & 2 & 1 & $?$ & 2 & 1 & 1 & 0 & 0 & 1 & 1 & 0 & 1 & 1 & 0 & 1 & 2 & 2 & 2 & 1 & 1 & 0 & 0 & ? & $?$ & 2 & 1 & & & & \\
\hline grupo Glyptodon & 3 & 1 & 3 & 3 & 1 & 1 & 2 & 1 & 1 & 1 & 1 & 1 & 1 & 1 & 1 & 2 & 2 & 2 & 1 & 1 & 1 & 1 & ( & 0 & 2 & 2 & & & 2 & \\
\hline grupo 1 & 3 & 1 & 3 & 3 & 1 & 1 & & 1 & 1 & 1 & 1 & 1 & & 1 & 1 & & 2 & & 1 & 1 & 1 & 1 & 0 & 0 & 2 & 2 & 2 & & 2 & \\
\hline
\end{tabular}

